\title{
BIRDS IN AND AROUND MACFAST CAMPUS, THIRUVALLA, KERALA
}

\section{P. M. NISHAD ${ }^{1} \&$ P. GREESHMA ${ }^{2}$}

${ }^{1}$ Department of Computer Applications, Macfast College, Thiruvalla, Pathanamthitta, Kerala

${ }^{2}$ Department of Wildlife Biology, Kerala Forest Research Institute, Peechi, Thrissur, Kerala

ABSTRACT

Avifaunal diversity of MACFAST campus and adjoining areas were carried out during March 2016 to June 2017. Ponds, grasslands, rooftops of buildings, playground and paddy fields were the major microhabitats of the study area. Birds were observed using Bushnell binocular (10x 50), spotting scope (10x-45x) and identified using physical features with the help of field guides and reference books. A total of 88 species from 12 orders and 38 families were recorded during the study and among these, Passeriformes and Pelicaniformes, dominated the list with 31 and 14 species, respectively. Three species of Near Threatened category (Mycteria leucocephala, Anhinga melanogaster, Threskiornis melanocephalus) and Ciconia episcopus from Vulnerable category were also recorded. Pompadour Green Pigeon Treron pompadora, Lesser coucal Centropus bengalensis, Pied Cuckoo Clamator jacobinus, Baillon's Crake Zapornia pusilla, White-browed Wagtail Motacilla maderaspatensis, Chestnut-tailed Starling Sturnia malabarica and Indian Paradise-flycatcher Terpsiphone paradise were the important sightings, during the survey. The presence of indigenous fruiting trees along with large trees as roosting sites, attract more birds to the campus.

KEYWORDS: Birds-Diversity- Macfast Campus-Thiruvalla-Kerala

Received: Sep 15, 2017, Accepted: Sep 30, 2017, Published: Oct 13, 2017, Paper Id: IJZROCT20173

\section{INTRODUCTION}

Birds are always a fascinating creature, which adds up the beauty of nature. Avifauna of MACFAST campus and adjoining areas were studied from March 2016 to June 2017. Mar Athanasios College for Advanced Studies (MACFAST) $\left(9^{0} 22^{\prime} 23^{\prime \prime} N \& 76^{0} 35^{\prime}\right.$ ' $\left.{ }^{\prime \prime} \mathrm{E}\right)$ in Pathanamthitta District is located about $3 \mathrm{~km}$ to the South of Thiruvalla town. The campus has varied micro habitats like ponds, grasslands, roof top of buildings, playground and paddy fields. The vegetation is dominated by Racosperma auriculiforme, Anacardium occidentale, Artocarpus heterophyllus, Racosperma mangium, Nephelium lappaceum, Artocarpus hirsutus, Ficus auriculata, Flacourtia jangomas,Mangifera indica, Bamboo Sps., Cocos nucifera, Azadirachta indica, Syzygium jambos, Phyllanthus emblica, Carica papaya, Cassia fistula, Sapindus emarginatus, Corypha umbraculifera, Lagerstroemia microcarpa, Tectona grandis, Macaranga indica, Bauhinia racemosa,, Albizia chinensis, Delonix regia, Muntingia calabura, Averrhoa carambola, Elaeocarpus serratus, Manilkara zapota and Artocarpus incises. Aquatic weeds like Eichhornia crassipes, Salvinia molesta were also present in water bodies near paddy fields.

\section{METHODOLOGY}

Birds were observed alone as well as in a team using Bushnell binocular (10 x 50), spotting scope (10 x$45 \mathrm{xs}$ ) and identified using physical features, with the help of field guides and reference books (Ali \& Ripley 1978; Grimmett et al. 2011). Observations were made from $06.00 \mathrm{hrs}$ to $10.00 \mathrm{hrs}$ and $16.00 \mathrm{hrs}$ to $19.00 \mathrm{hrs,}$ once in 
every week.

\section{RESULTS}

A total of 88 species from 12 orders and 38 families were recorded during the study (Table 1). Out of 500 species of Kerala (Praveen 2015), $17.6 \%$ of bird species were reported from this region. Of these 12 orders, Passeriformes, Pelicaniformes dominated the list with 31 and 14 species, respectively (Figure.1).

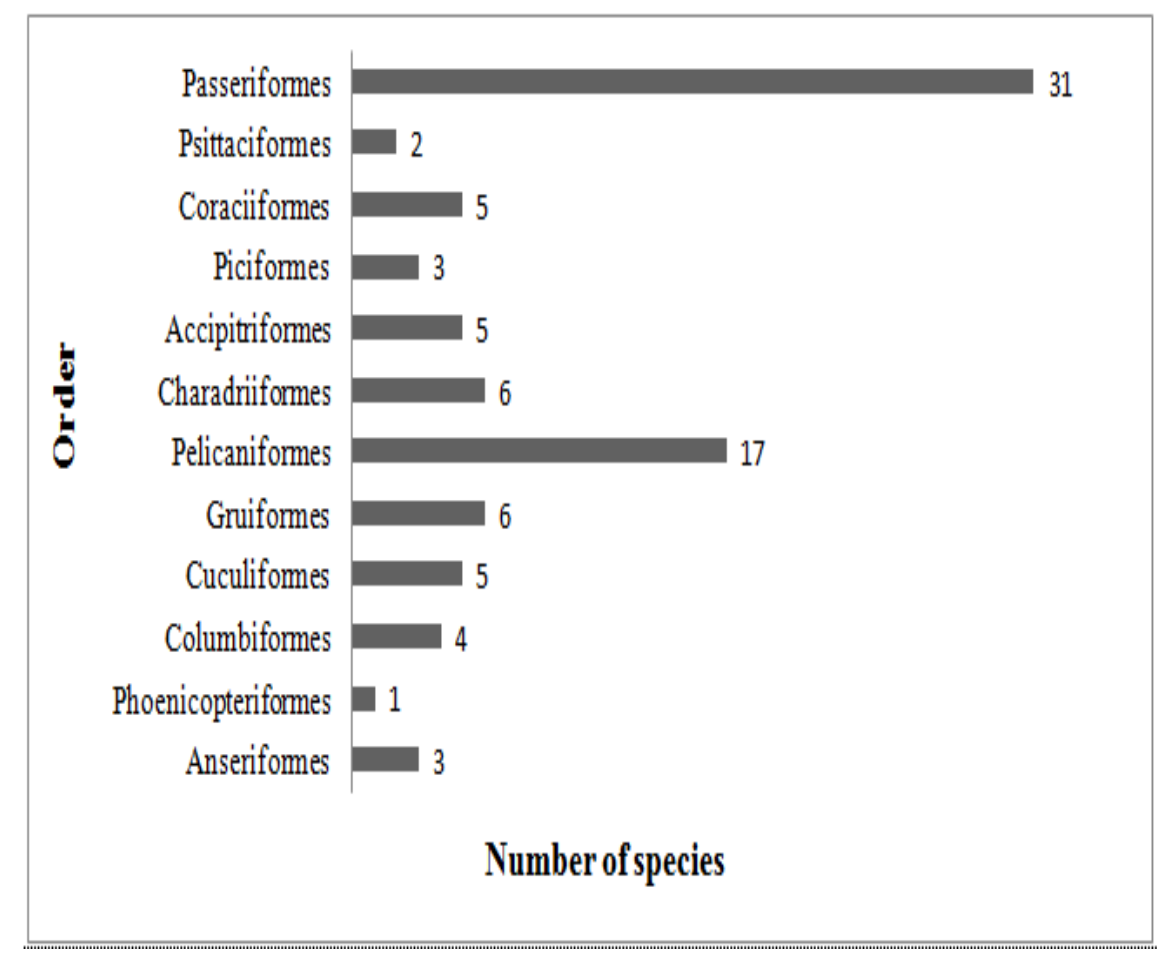

Figure 1: Number of Species Representing the Respective Orders from MACFAST Campus

Among the species recorded, $51 \%$ comprised of resident birds followed by $33 \%$ of local migrants and $16 \%$ of migratory birds. Of these, 47 species were wetland dependent birds and 41 species terrestrial birds. Little cormorant Microcarbo niger, Indian pond heron Ardeola grayii,, Grey-headed swamphen Porphyrio poliocephalus,, White-throated Kingfisher Halcyon smyrnensis, Black DrongoDicrurus macrocercus were the most abundantly seen resident birds. Three species of Near Threatened category (Mycteria leucocephala, Anhinga melanogaster, Threskiornis melanocephalus) and Woolly-necked Stork Ciconia episcopus, from Vulnerable category were also recorded. Pompadour Green Pigeon Treron pompadora,Lesser coucal Centropus bengalensis, Pied Cuckoo Clamator jacobinus, Baillon's Crake Zapornia pusilla, White-browed Wagtail Motacilla maderaspatensis, Chestnut-tailed Starling Sturnia malabarica, Indian Paradise-flycatcher Terpsiphone paradise were the important sightings. The paddy fields serve as an important foraging ground for several groups of birds like herons, ducks, cormorants, storks etc. Apart from foraging, hundreds of birds choose their resting place in this campus.

\section{DISCUSSION AND CONCLUSIONS}

During the study it was observed that the campus and adjoining areas had a rich variety of vegetation. The presence of indigenous fruiting trees, along with large trees as roosting sites, attract more birds to the campus. Least 
disturbances to the habitat and conservation awareness among the college people had led to the safe movement of birds all the time in the campus. Bird diversity at MACFAST campus and adjoining areas is unexplored yet and hence this work to record the avian diversity will form the base line information for future studies.

\section{REFERENCES}

1. Ali, S. \& S. D. Ripley.(1978). Hand book of the Birds of India and Pakistan. Oxford University Press, London, New York

2. Grimmet, R., C. Inskipp \& T. Inskipp. (2011). Birds of the Indian Subcontinent. Oxford University Press, Mumbai

3. Praveen, J (2015). A checklist of birds of Kerala, India. Journal of Threatened Taxa, 7(13): 7983-8009

\section{APPENDICES}

\section{ACKNOWLEDGEMENTS}

We are thankful to The Principal Rev. Fr. Pradeep Vazhatharamalayil and Mr. Pavin Thadathil, MACFAST College, for the infrastructural support. Our sincere gratitude to Mr. Rafy Kalletumkara and Mr. Praveen E. S. for helping in identification and Kerala State Council for Science Technology and Environment for providing financial aids.

Table 1: Checklist of Birds from MACFAST Collge Campus, Thiruvalla, Kerala

\begin{tabular}{|c|c|c|c|c|c|}
\hline Order & Family & Sl. No & Common Name & Scientific Name & IUCN \\
\hline \multirow{3}{*}{ Anseriformes } & \multirow{3}{*}{ Anatidae } & 1 & $\begin{array}{l}\text { Lesser Whistling- } \\
\text { Duck }\end{array}$ & Dendrocygna javanica & $\mathrm{LC}$ \\
\hline & & 2 & Cotton Pygmy-Goose & Nettapus coromandelianus & $\mathrm{LC}$ \\
\hline & & 3 & $\begin{array}{l}\text { Indian Spot-billed } \\
\text { Duck }\end{array}$ & Anas poecilorhyncha & $\mathrm{LC}$ \\
\hline Phoenicopteriformes & Podicipedidae & 4 & Little Grebe & Tachybaptus ruficollis & $\mathrm{LC}$ \\
\hline \multirow{4}{*}{ Columbiformes } & \multirow{4}{*}{ Columbidae } & 5 & Rock Pigeon & Columba livia & $\mathrm{LC}$ \\
\hline & & 6 & Spotted Dove & Streptopelia chinensis & $\mathrm{LC}$ \\
\hline & & 7 & Yellow-footed Pigeon & Treron phoenicopterus & $\mathrm{LC}$ \\
\hline & & 8 & $\begin{array}{l}\text { Pompadour Green } \\
\text { Pigeon }\end{array}$ & Treron pompadora & $\mathrm{LC}$ \\
\hline \multirow{5}{*}{ Cuculiformes } & \multirow{5}{*}{ Cuculidae } & 9 & Greater Coucal & Centropus sinensis & $\mathrm{LC}$ \\
\hline & & 10 & Lesser coucal & Centropus bengalensis & $\mathrm{LC}$ \\
\hline & & 11 & Pied Cuckoo & Clamator jacobinus & $\mathrm{LC}$ \\
\hline & & 12 & Asian Koel & Eudynamys scolopaceus & $\mathrm{LC}$ \\
\hline & & 13 & Indian Cuckoo & Cuculus micropterus & $\mathrm{LC}$ \\
\hline \multirow{6}{*}{ Gruiformes } & \multirow{6}{*}{ Rallidae } & 14 & $\begin{array}{l}\text { White-breasted } \\
\text { Waterhen }\end{array}$ & Amaurornis phoenicurus & $\mathrm{LC}$ \\
\hline & & 15 & Baillon's Crake & Zapornia pusilla & $\mathrm{LC}$ \\
\hline & & 16 & Watercock & Gallicrex cinerea & $\mathrm{LC}$ \\
\hline & & 17 & $\begin{array}{l}\text { Gray-headed } \\
\text { Swamphen }\end{array}$ & Porphyrio porphyrio & $\mathrm{LC}$ \\
\hline & & 18 & Common Moorhen & Gallinula chloropus & $\mathrm{LC}$ \\
\hline & & 19 & Common Coot & Fulica atra & $\mathrm{LC}$ \\
\hline \multirow{7}{*}{ elicaniformes } & \multirow{3}{*}{ Ciconiidae } & 20 & Asian Openbill & Anastomus oscitans & $\mathrm{LC}$ \\
\hline & & 21 & Woolly-necked Stork & Ciconia episcopus & VU \\
\hline & & 22 & Painted Stork & Mycteria leucocephala & NT \\
\hline & \multirow{4}{*}{ Ardeidae } & 23 & $\begin{array}{l}\text { Black-crowned Night } \\
\text { Heron }\end{array}$ & Nycticorax nycticorax & $\mathrm{LC}$ \\
\hline & & 24 & Grey Heron & Ardea cinerea & $\mathrm{LC}$ \\
\hline & & 25 & Purple Heron & Ardea purpurea & $\mathrm{LC}$ \\
\hline & & 26 & Great Egret & Ardea alba & $\mathrm{LC}$ \\
\hline
\end{tabular}




\begin{tabular}{|c|c|c|c|c|c|}
\hline & & 27 & Intermediate Egret & Ardea intermedia & $\mathrm{LC}$ \\
\hline & & 28 & Little Egret & Egretta garzetta & LC \\
\hline & & 29 & Western Reef-Heron & Egretta gularis & LC \\
\hline & & 30 & Cattle Egret & Bubulcus ibis & $\mathrm{LC}$ \\
\hline & & 31 & Indian Pond-Heron & Ardeola grayii & LC \\
\hline & & 32 & Glossy Ibis & Plegadis falcinellus & LC \\
\hline & $\begin{array}{l}\text { Threskiornithid } \\
\text { ae }\end{array}$ & 33 & Black-headed Ibis & $\begin{array}{l}\text { Threskiornis } \\
\text { melanocephalus }\end{array}$ & NT \\
\hline & Phalacrocoracid & 34 & Little Cormorant & Microcarbo niger & $\mathrm{LC}$ \\
\hline & & 35 & Indian Cormorant & Phalacrocorax fuscicollis & $\mathrm{LC}$ \\
\hline & Anhingidae & 36 & Oriental Darter & Anhinga melanogaster & NT \\
\hline & $\begin{array}{l}\text { Recurvirostrida } \\
\mathrm{e}\end{array}$ & 37 & Black-winged Stilt & Himantopus himantopus & $\mathrm{LC}$ \\
\hline & Charadriidae & 38 & Red-wattled Lapwing & Vanellus indicus & $\mathrm{LC}$ \\
\hline Charadriiformes & Jacanidae & 39 & Bronze-winged Jacana & Metopidius indicus & $\mathrm{LC}$ \\
\hline & & 40 & Common Snipe & Gallinago gallinago & LC \\
\hline & Scolopacidae & 41 & Marsh Sandpiper & Tringa stagnatilis & $\mathrm{LC}$ \\
\hline & & 42 & Wood Sandpiper & Tringa glareola & $\mathrm{LC}$ \\
\hline & & 43 & Crested Serpent Eagle & Spilornis cheela & $\mathrm{LC}$ \\
\hline & & 44 & $\begin{array}{l}\text { Eurasian Marsh- } \\
\text { Harrier }\end{array}$ & Circus aeruginosus & $\mathrm{LC}$ \\
\hline Accipitriformes & Accıpıtridae & 45 & Shikra & Accipiter badius & LC \\
\hline & & 46 & Brahminy Kite & Haliastur indus & $\mathrm{LC}$ \\
\hline & & 47 & Black Kite & Milvus migrans & $\mathrm{LC}$ \\
\hline & & 48 & $\begin{array}{l}\text { Common Golden- } \\
\text { backed Woodpecker }\end{array}$ & Dinopium javanense & LC \\
\hline Piciformes & Plcidae & 49 & $\begin{array}{l}\text { Lesser Golden-backed } \\
\text { Woodpecker }\end{array}$ & Dinopium benghalense & $\mathrm{LC}$ \\
\hline & Ramphastidae & 50 & White-cheeked Barbet & Psilopogon viridis & $\mathrm{LC}$ \\
\hline & Meropidae & 51 & Blue-tailed Bee-eater & Merops philippinus & $\mathrm{LC}$ \\
\hline & & 52 & Common Kingfisher & Alcedo atthis & $\mathrm{LC}$ \\
\hline Coraciiformes & & 53 & $\begin{array}{l}\text { Stork-billed } \\
\text { Kingfisher } \\
\end{array}$ & Pelargopsis capensis & $\mathrm{LC}$ \\
\hline & Alcedinıdae & 54 & $\begin{array}{l}\text { White-throated } \\
\text { Kingfisher }\end{array}$ & Halcyon smyrnensis & $\mathrm{LC}$ \\
\hline & & 55 & Pied Kingfisher & Ceryle rudis & $\mathrm{LC}$ \\
\hline Psittaciformes & Psittaculidae & 56 & Rose-ringed Parakeet & Psittacula krameri & LC \\
\hline Psittacilormes & Psittaculidae & 57 & Plum-headed Parakeet & Psittacula cyanocephala & $\mathrm{LC}$ \\
\hline & Artamidae & 58 & Ashy Woodswallow & Artamus fuscus & $\mathrm{LC}$ \\
\hline & Oriolidae & 59 & Indian Golden Oriole & Oriolus kundoo & $\mathrm{LC}$ \\
\hline & Uriondae & 60 & Black-hooded Oriole & Oriolus xanthornus & $\mathrm{LC}$ \\
\hline & & 61 & Black Drongo & Dicrurus macrocercus & $\mathrm{LC}$ \\
\hline & Dicruridae & 62 & $\begin{array}{l}\text { Greater Racket-tailed } \\
\text { Drongo }\end{array}$ & Dicrurus paradiseus & $\mathrm{LC}$ \\
\hline & Aegithinidae & 63 & Common Iora & Aegithina tiphia & $\mathrm{LC}$ \\
\hline & & 64 & Rufous Treepie & Dendrocitta vagabunda & $\mathrm{LC}$ \\
\hline Passeriformes & Corvidae & 65 & House Crow & Corvus splendens & LC \\
\hline & Monarchidae & 66 & $\begin{array}{l}\text { Indian Paradise- } \\
\text { flycatcher }\end{array}$ & Terpsiphone paradisi & $\mathrm{LC}$ \\
\hline & Dicaeidae & 67 & $\begin{array}{l}\text { Pale-billed } \\
\text { Flowerpecker }\end{array}$ & Dicaeum erythrorhynchos & $\mathrm{LC}$ \\
\hline & & 68 & $\begin{array}{l}\text { Purple-rumped } \\
\text { Sunbird }\end{array}$ & Leptocoma zeylonica & LC \\
\hline & Nectarını1ıae & 69 & Little Spiderhunter & Arachnothera longirostra & LC \\
\hline & & 70 & Loten's Sunbird & Cinnyris lotenius & LC \\
\hline
\end{tabular}




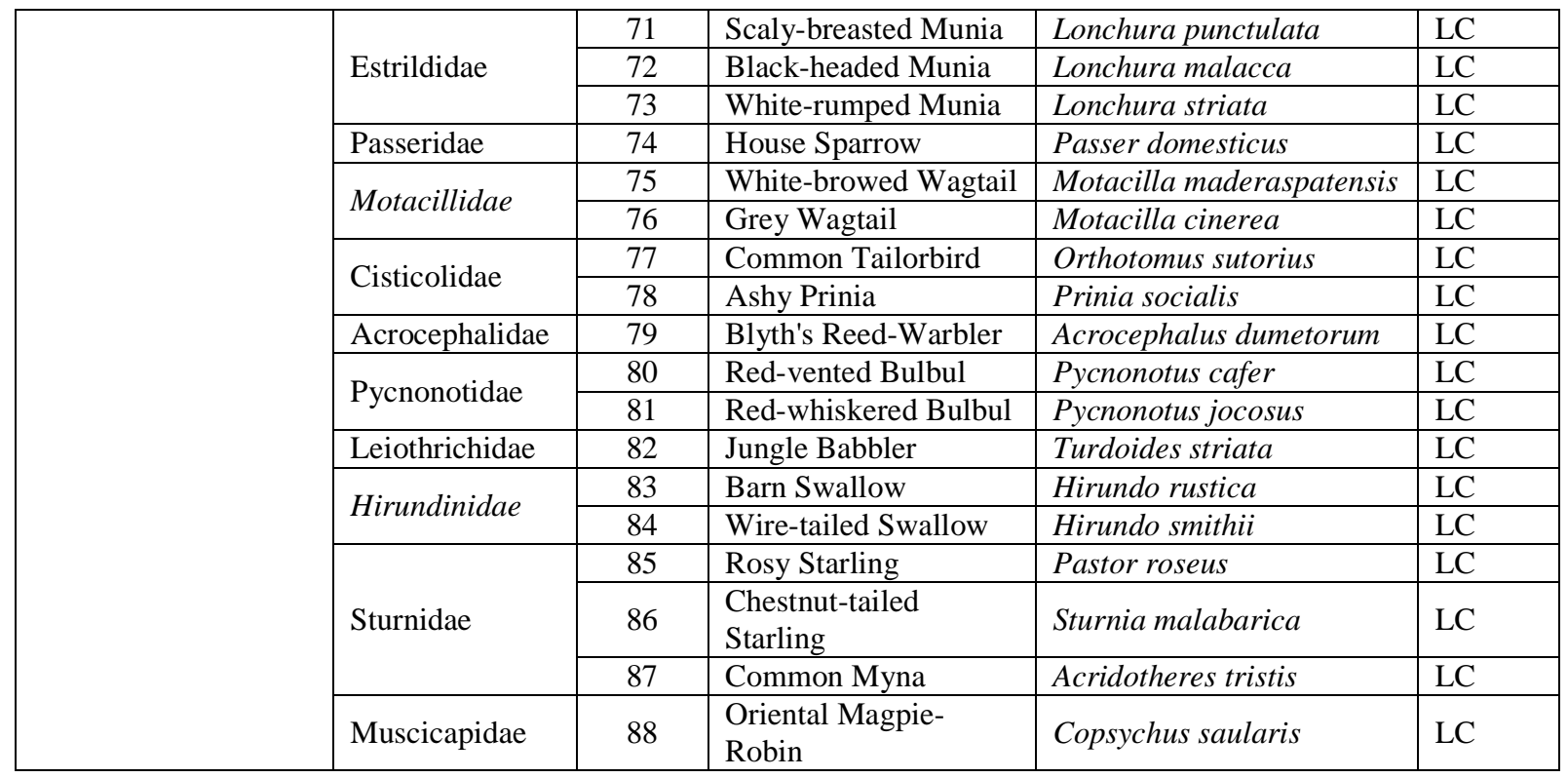


